\title{
Robot-assisted laparoscopic myomectomy: current status
}

This article was published in the following Dove Press journal:

Robotic Surgery: Research and Reviews

23 January 2017

Number of times this article has been viewed

\author{
Sara E Arian \\ Jessian L Munoz \\ Suejin Kim \\ Tommaso Falcone \\ Department of Obstetrics, \\ Gynecology, and Women's Health \\ Institute, Cleveland Clinic, Cleveland, \\ $\mathrm{OH}$, USA
}

\begin{abstract}
Robotic-assisted surgery has seen a rapid development and integration in the field of gynecology. Since the approval of the use of robot for gynecological surgery and considering its several advantages over conventional laparoscopy, it has been widely incorporated especially in the field of reproductive surgery. Uterine fibroids are the most common benign tumors of the female reproductive tract. Many reproductive-aged women with this condition demand uterine-sparing surgery to preserve their fertility. Myomectomy, the surgical excision of uterine fibroids, remains the only surgical management option for fibroids that entails preservation of fertility. In this review, we focus on the role of robotic-assisted laparoscopic myomectomy and its current status, in comparison with other alternative approaches for myomectomy, including open, hysteroscopic, and traditional laparoscopic techniques. Several different surgical techniques have been demonstrated for robotic myomectomy. This review endeavors to share and describe our surgical experience of using the standard laparoscopic equipment for roboticassisted myomectomy, together with the da Vinci Robot system. For the ideal surgical candidate, robotic-assisted myomectomy is a safe minimally invasive surgical procedure that can be offered as an alternative to open surgery. The advantages of using the robot system compared to open myomectomy include a shorter length of hospital stay, less postoperative pain and analgesic use, faster return to normal activities, more rapid return of the bowel function, and enhanced cosmetic results due to smaller skin incision sizes. Some of the disadvantages of this technique include high costs of the robotic surgical system and equipment, the steep learning curve of this novel system, and prolonged operative and anesthesia times. Robotic technology is a novel and innovative minimally invasive approach with demonstrated feasibility in gynecological and reproductive surgery. This technology is expected to take the lead in gynecological surgery in the upcoming decade.
\end{abstract}

Keywords: myomectomy, uterine fibroid, robotic surgery, robotic-assisted laparoscopic myomectomy

\section{Introduction}

The use of the robot was first reported in gynecological surgery in 2000 by Falcone et al. ${ }^{1}$ Robotic-assisted surgeries in gynecology have since expanded to include hysterectomy, myomectomy, salpingo-oophorectomy, resection of endometriosis, tubal reanastomosis, and more specialized surgeries, such as sacrocolpopexy and lymphadenectomy. ${ }^{2}$ Robotic surgery has been readily accepted in gynecological oncology surgery education. A survey in 2010 showed that $95 \%$ of gynecologic oncology fellowship programs
Department of Obstetrics, Gynecology, and Women's Health Institute, Cleveland Clinic, 9500 Euclid Avenue, Cleveland, $\mathrm{OH} 44195$, USA

Tel +l 7l46566877

Email sarian@bcm.edu 
use the robot for surgical procedures and $94 \%$ of graduating fellows planned to perform robotic surgery in their future careers. ${ }^{3}$ Compared to laparotomy, robotic-assisted surgery offers many of the same postoperative advantages as traditional minimally invasive laparoscopy. These advantages include excellent cosmesis from smaller incisions, minimal blood loss, lower infection rates, less postoperative pain, and shorter length of hospital stay. ${ }^{4}$ Advantages of robotic surgery over conventional laparoscopy mainly lie in the engineering of the robot system that allows for greater wrist mobility, thus allowing the surgeon to execute more complex tasks, such as delicate tissue dissection and intracorporeal knot tying. ${ }^{5,6}$

\section{The use of robot in benign gynecology Robotic-assisted laparoscopic hysterectomy}

Hysterectomy continues to remain the most common major gynecological surgery. Over time, various approaches to hysterectomy have developed, including open abdominal (TAH), vaginal (TVH), laparoscopic (TLH), robotic-assisted (RA-TLH), and the hybrid laparoscopic-assisted vaginal hysterectomy (LAVH). The trend over time has been to avoid open abdominal surgery and shift focus to minimally invasive approaches. In a 2004 position statement, the American Association of Gynecologic Laparoscopists (AAGL) stated that hysterectomies (for benign disease) should be approached vaginally or via minimally invasive techniques when possible. ${ }^{7}$

Performance of robotic-assisted laparoscopic hysterectomy (RA-TLH) has become very frequent for benign disease despite the steep learning curve. Wright and colleagues assessed RA-TLH trends nationally and noted a 19-fold increase in the rates of RA-TLH over a 4-year period. ${ }^{8}$ In addition, these authors noted that although the length of hospital stay was shortened and postoperative blood transfusion rate was reduced, the overall cost of RA-TLH was still higher than that of TLH. The operative time has also been noted to be longer with RA-TLH. A Cochrane review in 2014 also concluded that RA-TLH was comparable to TLH with respect to blood loss and safety, but there was a significant increase in the overall cost of surgery. ${ }^{9}$

\section{Robotic excision of endometriosis}

Resection of endometriosis is one of the most difficult laparoscopic surgeries. This is mainly due to the inherent nature of the disease, which results in significant scarring, distortion of the anatomy, loss of surgical planes, and immobility of pelvic organs. ${ }^{10}$ Robotic assistance and better visualization are meant to enhance the resection outcomes of this complex surgery. Current data, however, does not support this statement. In a direct comparison of robotic-assisted laparoscopy with traditional laparoscopy, specifically for endometriosis, Nezhat et al found no significant differences in the amount of blood loss, conversion rate, complications, and duration of hospital stay between the two techniques. The only difference noted was longer average operative time associated with the robotic approach. ${ }^{11}$ As the overall outcomes of surgery do not appear to differ between robotic-assisted and traditional laparoscopy, the greatest advantage is noted in the complexity of the cases achieved with robotic assistance. Resection of deep infiltrating endometriosis involving parts of the rectum, sigmoid colon, and extensive ureterolysis can all be achieved with the assistance of the robot system. ${ }^{12}$ Studies have shown that with robotic assistance, these surgeries can be performed safely and effectively. In addition, Neme et al showed not only feasibility and safety of the robot system, but also reported subsequent fertility in 4 of 10 patients in these series. ${ }^{12}$

\section{Robotic tubal reanastomosis}

Permanent female sterilization via fallopian tubal ligation remains a common method of contraception. Tubal reanastomosis has traditionally been performed by laparotomy with varying success in fertility and the risk for ectopic pregnancy. ${ }^{13}$ Given the overall advantages in postoperative care, the laparoscopic technique for tubal reanastomosis has now developed into a viable option. The difficulty with the laparoscopic approach arises from the microsurgery technique and the need for precision and meticulousness, thus resulting in a steep learning curve.

Since robotic assistance allows for greater control, visualization, and movement, studies have been performed to assess the feasibility and efficacy of robotic approaches for tubal reanastomosis. Rodgers et al compared the robotic approach with mini-laparotomy. ${ }^{14}$ The operative time was again noted to be longer, and the costs were estimated to be higher with the robotic approach, but return to full function was significantly shorter compared to mini-laparotomy (1 week sooner). In addition, Patel et al compared robotic tubal reanastomosis to the laparotomy approach in their study. ${ }^{15}$ The conclusion of this study was that while the operative time was longer with the robot, the mean hospital stay was significantly shorter (4 hours vs 35 hours), thus making the robotic approach feasible and comparable in terms of cost. Pregnancy rates were also similar with both techniques (28\% vs $30 \%)$, with 
a mean time from sterilization to reversal being 8.5 years (robot: 8.1 years, open: 7.8 years, $P=0.23$ ).

\section{Uterine leiomyoma}

Uterine leiomyoma, also known as uterine fibroid, is the most common benign gynecologic neoplasm during the reproductive years with a large range of prevalence from $5.4 \%$ to $77 \% .{ }^{16}$ It is considered to be a benign overgrowth of the myometrium, which predominantly consists of smooth muscle cells and fibrous tissue, encased in a pseudocapsule rich in collagen. Different types of uterine fibroids include intracavitary, submucosal, intramural, subserosal, and pedunculated fibroids. Even though uterine fibroids may be asymptomatic, patients commonly report pelvic pain during or outside of menstrual cycles, excessive menstrual blood loss, pressure symptoms including urinary and bowel habit changes, and infertility. As a result, uterine fibroids are the most common indication for hysterectomy in the United States and have an enormous impact on the healthcare costs and quality of life. ${ }^{16,17}$

Management options for fibroids include medical and surgical approaches. Medical therapies include non-hormonal therapies, such as non-steroidal anti-inflammatory drugs and tranexamic acid, and hormonal therapies, including oral contraceptive pills, gonadotropin $(\mathrm{GnRH})$ analogs, progestinimpregnated intrauterine system, selective estrogen receptor modulators, antiprogestin drugs, selective progesterone receptor modulators, androgens, and aromatase inhibitors. ${ }^{17,18}$ Non-hormonal therapies mainly serve to treat the symptoms of bleeding, and some of the hormonal therapies aim to temporarily decrease the size of the fibroids; however, these options may also be used as an adjunct to surgical therapy and for those who are approaching menopause and desiring to avoid surgical intervention. ${ }^{17-19}$ Surgical approaches addressing uterine fibroids include uterine artery embolization, myomectomy, and hysterectomy. Uterine artery embolization appears to be effective for symptomatic relief; however, there are complications associated with this procedure including possible impaired fertility due to compromised ovarian blood supply and possible poor obstetrical outcomes including preterm labor. Approximately $15 \%-32 \%$ of these patients will require further surgical intervention within 2 years of the procedure, and $35 \%$ will end up with a hysterectomy in 10 years. ${ }^{20,21}$ In patients attempting to conceive, it is typically recommended to consider removal of submucosal fibroids as they have a negative impact on fertility. Intramural uterine fibroids that distort the uterine cavity or larger intramural fibroids greater than $4 \mathrm{~cm}$ in size even without distorting the uterine cavity may also negatively affect fertility and therefore their removal should be considered. ${ }^{22,23}$ Hysterectomy is the definitive surgical management for myomas; however, myomectomy should be considered prior to proceeding to hysterectomy, in order to decrease postoperative morbidity and also in the cases where fertility preservation is desired. ${ }^{20}$

\section{Surgical techniques for myomectomy Abdominal myomectomy}

Abdominal myomectomy, also known as open myomectomy, may be considered when the uterus is significantly enlarged, typically above the level of the umbilicus, and/or when multiple myomas are present. The surgical approach to myomectomy however, should be highly individualized, taking into consideration other factors, such as prior surgical history and patient's comorbid conditions. ${ }^{24}$ Some of the advantages of abdominal myomectomy include no limitations on the size and the number of fibroids to be removed, the ability to palpate and completely remove all the myomas, and perhaps, ease of the open surgery technique depending on the surgeon's experience and skills. ${ }^{25}$ Abdominal myomectomies are carried out with a transverse suprapubic incision if feasible; however, a vertical incision may be needed for access depending on the case and the size of the uterus. ${ }^{25}$ The uterus is initially exteriorized with or without the aid of myoma screws. ${ }^{25-27}$ Abdominal contents are packed with laparotomy sponges and retractors may be used. Several approaches have been proposed to minimize intraoperative blood loss; of these, physical occlusion of the blood flow by using tourniquets, uterine and ovarian artery ligation, and pre-myomectomy uterine artery embolization may be helpful in certain patients. ${ }^{25}$ According to a study by Taylor et al, there may be an advantage in employing tourniquets at the level of the cervix and bilateral infundibulopelvic ligaments. This technique has been shown to be associated with significantly decreased blood loss and transfusion rates. ${ }^{28}$ This technique along with vasopressin injection may be considered prior to proceeding with the actual removal of fibroids in order to improve surgical blood loss. ${ }^{29}$ After securing the tourniquets, diluted vasopressin in normal saline is injected into the myometrium surrounding the fibroid to decrease intraoperative blood loss via vasoconstriction. ${ }^{25-27} \mathrm{~A}$ small incision is made over the area overlying the bulk of the fibroid taking care to avoid the cornua and tubal ostia in order to minimize uterine damage, as well as to avoid posterior uterine incisions if possible to decrease the risk of adhesion formation. Multiple fibroids are usually attempted to be removed 
through this single incision if possible. ${ }^{26,27}$ After identification of the fibroid capsule, it should be enucleated using a myoma screw or single-tooth tenaculum for traction, with sharp dissections as needed using Metzenbaum scissors or electrocautery. ${ }^{30}$ It is essential to avoid excessive traction on the myoma bed and instead to push the myometrial and endometrial fibroids down and away in order to prevent breaching of the endometrial cavity. Blood vessels are usually located at the base of the myoma and their entry should be avoided if possible. The closure of the defect should be performed from the deepest layer to obtain hemostasis while including all myometrial tissue to allow for optimal tissue healing and remodeling. The myometrial layer can be re-approximated in multiple layers using size 0 or 1 absorbable polyglactin sutures in a simple interrupted or a running fashion. After complete closure of the dead space, the serosal layer may be closed with size 3-0 or 4-0 delayed absorbable or poliglecaprone suture in a subcuticular fashion followed by burying of the knot. Of note, it is imperative to have excellent hemostasis all throughout the surgical procedure and keep the serosal layer moist to ensure that the least amount of adhesions will form. A free omental graft can also be utilized to help with adhesion prevention.

\section{Laparoscopic myomectomy}

Laparoscopic myomectomy may be considered when there is a single intramural or serosal fibroid of $\leq 15 \mathrm{~cm}$ size or $\leq 3$ fibroids each $<5 \mathrm{~cm}$ in diameter. However, this surgical route is dependent on the surgeon's expertise and may also be performed in patients with myomas $>20 \mathrm{~cm}$ in diameter. ${ }^{31}$ According to a study by Sinha et al, for an experienced surgeon, there appears to be no limitations to the number, size, and location of the myomas to perform laparoscopic myomectomy. ${ }^{32}$

The first step is to place a uterine manipulator. ${ }^{33}$ Trocars are then inserted based on the size and location of the myomas noted from the imaging studies and physical examination prior to the actual surgery. A supraumbilical skin incision can be made between the xiphoid process and the umbilicus, also known as Lee-Huang point, or alternatively at Palmer's point, to accommodate for the entry site. Accessory trocars may be placed in suprapubic positions lateral to the inferior epigastric blood vessels. As with abdominal myomectomy, vasopressin can be injected into the myometrial layer surrounding the fibroid using a laparoscopic needle. This is followed by creating a vertical uterine incision using the unipolar hook on high cutting current down to the pseudocapsule to help with suturing, which can be an elliptical or a transverse incision depending on the location and the size of the myoma. The exposed fibroid is then enucleated by placing traction and grasping on to the myoma with a grasping forceps or by using a drill and placing counter traction on the uterine manipulator and/or pulling the edges of the hysterotomy incision. A unipolar hook can be used to dissect off the pseudocapsule attachments. After removal of the fibroid, uterine cavity must be checked for any breaches of its integrity. If the cavity has been entered, the endometrium should be re-approximated suing a 3-0 polyglactin suture. ${ }^{31}$ Interrupted or running sutures starting from the deepest myometrial layer are then placed as with the abdominal approach. Using a barbed suture may be of great advantage here for appropriate tension distribution and possibly shortening the operative time by eliminating the need for laparoscopic suturing. The specimen can be placed inside a bag for in-bag morcellation, or alternatively, can be extracted via a mini-laparotomy incision, which is usually $<4 \mathrm{~cm}$ in size. ${ }^{34}$ Of note, these incisions may be associated with more postoperative pain, increased blood loss, and longer hospital stay compared to the laparoscopic approach without similar skin incisions.

\section{Comparison of open and minimally invasive myomectomy}

Multiple studies have compared the surgical outcomes of different approaches of myomectomies. Holzer et al investigated postoperative pain within 72 hours of surgery in a doubleblinded prospective trial comparing laparoscopy to abdominal myomectomy, with the results favoring the laparoscopy group. ${ }^{35}$ Cicinelli et al conducted a prospective randomized study comparing laparoscopic myomectomy to myomectomy via mini-laparotomy for removal of one to three myomas $<7 \mathrm{~cm}$, and showed better outcomes in the laparoscopic myomectomy group in terms of the length of hospital stay, the amount of blood loss, and postoperative ileus, without an increase in operative time. ${ }^{36}$ In a meta-analysis comparing laparoscopic to open myomectomy, laparoscopic approach was noted to be associated with less amount of blood loss, less need for blood transfusion, less postoperative pain, and a shorter hospital stay. The operative time, however, was noted to be longer with laparoscopy, which was concluded to be due to the learning curve the surgeons had to experience with this approach. ${ }^{37} \mathrm{~A}$ recent retrospective review of open versus laparoscopic myomectomy from Taiwan showed decreased blood loss, transfusion rates, and shorter operative time in the laparoscopic myomectomy group; however, a slightly higher surgical cost. ${ }^{38,39}$ In the Cochrane review by Bhave et al comparing open to minimally invasive myomectomies, 
post-operative pain was noted to have inconsistent results between the two groups at 48 hours and 72 hours after surgery. This was reported to be likely due to mini-laparotomy incisions that were included in some of the studies of the laparoscopic approach. ${ }^{40}$ Postoperative febrile morbidity and intraoperative blood loss were both noted to be less common in laparoscopic compared to open approaches. ${ }^{40}$

Open myomectomy has been compared not only to laparoscopic but also to robotic myomectomy. Bakarat et al retrospectively compared abdominal myomectomy to laparoscopic and robotic myomectomies. ${ }^{41}$ In this study, it was noted that the myomas were significantly larger in the open and the robotic groups compared to the laparoscopic group. Surgical time was reported to be longer in the robotic compared to the open group, without any differences observed between the robotic and laparoscopic groups or between laparoscopic and open approaches. Blood loss was noted to be more significant in the open group without any differences noted between the robotic and laparoscopic approaches. Hospital stay was shorter in the robotic group compared to the open approaches, which made up for the longer operative time. In a recent meta-analysis of robotic versus laparoscopic and open myomectomies, the operative time was noted to be shorter for open compared to minimally invasive approaches $(95 \%$ confidence interval $[\mathrm{CI}]$ : 60.41-109.29), with no differences noted between these two approaches. The amount of blood loss was less in minimally invasive approaches without any difference between robotic and laparoscopic techniques $(42.10 \mathrm{~mL} /$ operation; $95 \%$ CI: 1.28-85.48). The rate of blood transfusion was higher (981 patients, odds ratio [OR], 0.20; 95\% CI: 0.09-0.43) and the length of hospital stay was longer (95\% CI: 1.40-2.29) in the open group without any observed differences in the other two groups (870 patients, OR: 1.13; 95\% CI: 0.42-3.07 for blood transfusion, 0.04 days/patient; 95\% CI: 0.09-0.18 for the length of hospital stay). Finally, no difference was detected in postoperative pain levels between robotic and open approaches. ${ }^{42}$ In another study with approximately 40 cases in each group, robotic and laparoscopic approaches were compared and again no difference was observed in short-term surgical outcomes, including operative time, blood loss, and postoperative complications between the two. ${ }^{43}$ A small study by Nezhat et al also compared 15 robotic to 35 laparoscopic cases and showed longer operative time in the robotic group; however, there were no differences noted in the amount of blood loss, the length of hospital stay, and postoperative complications between the two approaches. ${ }^{44}$ These results should be considered when choosing the optimal route of myomectomy and ultimately the decision should be individualized based on each patient.

\section{Robotic-assisted laparoscopic myomectomy Indications, patient selection, and preoperative preparation}

Robotic-assisted myomectomy is a commonly performed reproductive surgery. As previously mentioned, in order to be able to determine the most appropriate route for surgical management of uterine leiomyoma, having the principle knowledge of anatomic factors is essential. Some of these key factors include myoma size, extent, number, location, and proximity to the uterine cavity ${ }^{45}$ Selection of the appropriate patients who would be ideal surgical candidates for robotic myomectomy is an important initial step in surgical planning for uterine fibroids. Choosing the robotic approach versus other surgical routes for myomectomy should therefore be individualized and based on the appropriate patient selection, as previously discussed. Individual's medical and surgical histories are also factors that should be considered. Other principles that must be taken into consideration prior to choosing a surgical approach for myomectomy include surgeon's level of training and experience, and the availability and costs of surgical equipment. In general, the most suitable candidates for robotic approach are those with subserosal, intramural, fundal, or pedunculated fibroids. ${ }^{45}$ Some women may not be considered as appropriate candidates for robotic myomectomy. These include patients with an enlarged uterus $>16$ weeks in size, those with more than a total of five myomas present in the uterus, and those with uterine fibroids located in anatomically challenging locations, such as those adjacent to the broad ligament, cervix, uterine cornua, or uterine blood vessels. Robotic myomectomy may also not be suitable when there is a single uterine fibroid present, which is $>15 \mathrm{~cm}$ in size. ${ }^{40,46}$ Additionally, fibroids abutting the endometrial cavity or fibroids with a submucousal component may not be ideal for the robotic approach. Removal of these fibroids can be more challenging and associated with a higher rate of entering the endometrial cavity. Closure of the cavity defect may be more complicated with the use of the robot given the lack of tactile feedback. ${ }^{47}$ It is important to note that robotic myomectomy may be attempted in these cases, as long as the patient is made aware that conversion to open surgery can be anticipated at a higher rate. The general conversion rate of a robotic-assisted myomectomy to laparotomy is $\sim 11.3 \%{ }^{48}$ This rate is similar to that of conversion in laparoscopic 
myomectomy. The conversion rate may also vary based on the surgeon's experience and patient selection. The maximum size and number of uterine fibroids that are safely amenable to robotic-assisted laparoscopic myomectomy must therefore be individualized to each surgeon based on their expertise, training, and their level of comfort.

Once the surgical technique has been selected as roboticassisted myomectomy, the same preoperative preparation will be implemented as in open, laparoscopic, and hysteroscopic approaches. If the surgical candidate is anemic, pretreatment with a GnRH-releasing hormone agonist should be considered to address anemia and also to help reduce the uterine or fibroid volume. ${ }^{49}$ In our practice, we also consider obtaining preoperative magnetic resonance imaging (MRI) for all myomectomy candidates, in order to delineate the uterine dimensions and the number, size, and the exact location of the uterine fibroids. MRI can also help in differentiating uterine fibroids from adenomyosis with a high specificity for diagnosis of adenomyosis. ${ }^{50} \mathrm{~A}$ preoperative diagnosis of adenomyosis is of extreme importance, as it can affect the entire surgical approach. In our practice, MRI is therefore also obtained in the cases of suspected adenomyosis or when the diagnosis is unclear.

\section{Surgical technique for robotic myomectomy}

Multiple variations in surgical techniques for robotic myomectomy have been described. In this section, we are going to discuss the technique that is typically used at our institution for robotic-assisted myomectomy.

We use the standard laparoscopic equipment for our robotic-assisted myomectomies, along with the da Vinci Robot system with the addition of the patient-side robot, the vision cart, and the robot operator console. The patient is positioned similar to a conventional laparoscopy surgery in a dorsal lithotomy position, with arms tucked on the sides in a neutral position. Although some recommend the routine use of maximum Trendelenburg position for robotic surgery, we typically only use as much Trendelenburg positioning as needed and is tolerated by the patient to be able to perform the surgery. Once the patient has been prepped and draped as for a conventional gynecological laparoscopy, a uterine manipulator is inserted inside the uterus. This manipulator is placed to optimize the exposure for enucleation of the myoma as well as to provide a means of instilling dye into the uterus, in order to identify and avoid breaching of the endometrial cavity during the surgery. Chromopertubation using the uterine manipulator will also allow for confirmation of tubal patency. We typically use RUMI (CooperSurgical) as a uterine manipulator. Once the manipulator is securely inserted inside the uterus, a foley catheter is then placed to drain the bladder. We typically start the case with conventional laparoscopy to confirm that there are no contraindications to the use of the robot. Given the recent controversy regarding the use of the power morcellator, we initially start with placing a GelPoint suprapubically. Approximately $2 \mathrm{~cm}$ superior to symphysis pubis, a small $3-4 \mathrm{~cm}$ low transverse skin incision will be made and carried down to the underlying layer of fascia. The fascia is then incised in the midline and extended bilaterally. After dissection of the fascia from the rectus muscles, the peritoneal cavity is entered in a usual fashion, and the GelPoint will be inserted and the abdomen will be insufflated. This suprapubic incision is not large and and not cosmetically disturbing to the patient, as it is made within the natural hairline. As with any minimally invasive technique, trocar placement is important in order to avoid contact between robotic arms. Laparoscopic trocars are then placed as follows:

Prior to insertion of each trocar, $0.25 \%$ Bupivicaine is injected subcutaneously. Initially, a small $5 \mathrm{~mm}$ infraumbilical skin incision is made with a scalpel and the umbilical trocar is placed. This $5 \mathrm{~mm}$ trocar is initially placed to perform a laparoscopic survey and to guarantee that the procedure can be performed robotically. An optical trocar is then used to enter the abdominal cavity. After performing a thorough survey of the abdominal and pelvic cavities and confirming that a robotic approach is appropriate, we then enlarge the $5 \mathrm{~mm}$ trocar to $12 \mathrm{~mm}$ which can be used to place the robotic camera. Next, two $8 \mathrm{~mm}$ robotic trocars are placed at an angle $\sim 15^{\circ}$ inferiorly and $8-12 \mathrm{~cm}$ laterally to the umbilical port, in order to accommodate the robotic arms without any difficulty. The ports can be placed more cephalad in the cases of large uterine fibroids. We then place a $5 \mathrm{~mm}$ accessory port typically in the left upper quadrant at the Palmer's point. Through this port, a $5 \mathrm{~mm}$ laparoscopic tenaculum forceps can be used to assist with the enucleation of leiomyoma. All of these ports are placed under direct visualization. The GelPoint is used both to pass needles and also for tissue extraction at the end of the case. If a suprapubic incision is not desirable for the patient, the left upper quadrant accessory port can be used to pass and handle needles, and the GelPoint can be placed inside the larger umbilical incision for tissue extraction at the conclusion of the case. After placing the trocars, we then "side dock" the da Vinci Robot, allowing more space for an assistant to provide uterine manipulation. As previously 
explained, the patient will be placed in the Trendelenburg position as tolerated. The robot can be placed either between each of the stirrups, in between the patient's legs, or on the patient's side. With our experience, the third robotic arm is rarely needed, and we therefore elect not to use it. Once all the robotic arms are docked, the robotic camera and instruments are inserted into their appropriate trocars. Our preferred set of instruments include the tenaculum forceps, Harmonic device, Maryland bipolar forceps connected to cautery, and two needle drivers. We then proceed with the actual surgical procedure as described below in detail.

Prior to making the hysterotomy, we perform a serosal injection with a solution of dilute vasopressin (20 units of vasopressin in $200 \mathrm{~mL}$ of normal saline). This solution is injected into the serosa and the myometrial layer surrounding the fibroid. A laparoscopic needle tip device is attached to a syringe containing the dilute vasopressin and is used for injection. If the correct planes are injected, the uterus will start becoming blanched. Vasopressin can be rarely associated with severe cardiopulmonary complications, including bradycardia, hypertension, and cardiac arrest, even in healthy individuals and should be used with caution. ${ }^{51-54}$ The use of vasopressin must therefore be avoided in individuals with coronary artery disease or high blood pressure, in order to prevent potential lethal complications. Some other agents that have been successfully described in the literature to decrease blood loss during myomectomy are preoperative misoprostol, bupivacaine with epinephrine, tranexamic acid, and the use of uterine tourniquets. ${ }^{55}$ Some of the other techniques that have been reported to assist with decreased blood loss include ligation of the uterine arteries temporarily or permanently prior to myomectomy. Temporary occlusion of the uterine arteries can be achieved using catheters, elastic tourniquets, or rubber clamps that are wrapped around the uterine arteries. After injection with vasopressin, hysterotomy can be made through the serosa and the myometrium using a Harmonic device until the fibroid capsule is reached and opened. Compared to the use of electrosurgery and epinephrine, the use of harmonic scalpel has shown a decrease in both operative time and blood loss in a randomized, controlled trial. ${ }^{56} \mathrm{We}$ prefer to make the hysterotomy horizontally if possible to facilitate suturing. With the advantage of the robot in providing the ability to maneuver the instruments easier than traditional laparoscopy, hysterotomy and suturing can also be performed in a vertical fashion (Figure 1). Prior to making the hysterotomy, attention should be paid to the exact location of the fallopian tubes, ovaries, cornua, and the uterine vessels in order to stay distant from these important anatomical landmarks. Hemostasis can be maintained using the Maryland bipolar electrocautery throughout making the hysterotomy (Figure 2). Excessive coagulation, however, may compromise the integrity of the myometrium and make closure of the hysterotomy more difficult. Some of the hemostatic agents that are commonly used in our practice are Surgiflo (Ethicon Inc.) and Floseal (Baxter, Deerfield IL, USA). These are both thrombin-infused gelatin products that can be directly applied on the bleeding areas. ${ }^{57}$ After entering the capsule of the fibroid and exposing the myoma fibers, a robotic tenaculum is placed on the fibroid, and it is enucleated using blunt dissection along with the use of harmonic energy as needed (Figures 3 and 4). Careful attention should be paid in order to avoid putting extra traction on the myoma, as breaching of the endometrial cavity can occur as a result of avulsing myoma, and this can ultimately compromise fertility. Preservation of the endometrial cavity is of extreme importance for the reproductive surgeon, especially when the end point of myoma surgery is fertility preservation. Chromopertubation with diluted methylene blue can be used to confirm the integrity of the endometrial cavity throughout the surgery. Pedunculated fibroids are removed in a similar fashion.

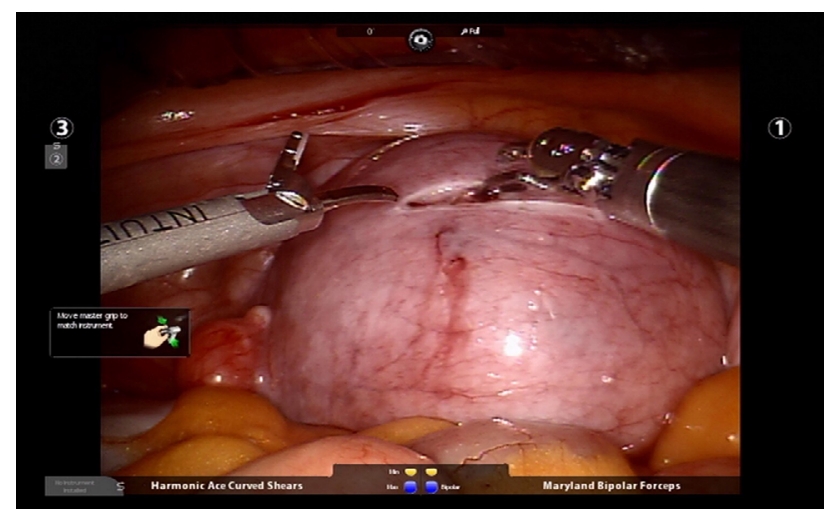

Figure I Hysterotomy is made horizontally through the serosa and the myometrium using a Harmonic device.

Note: Photo courtesy of Dr Tommaso Falcone, Department of Obstetrics, Gynecology, and Women's Health Institute, Cleveland Clinic, Cleveland, Ohio, USA.

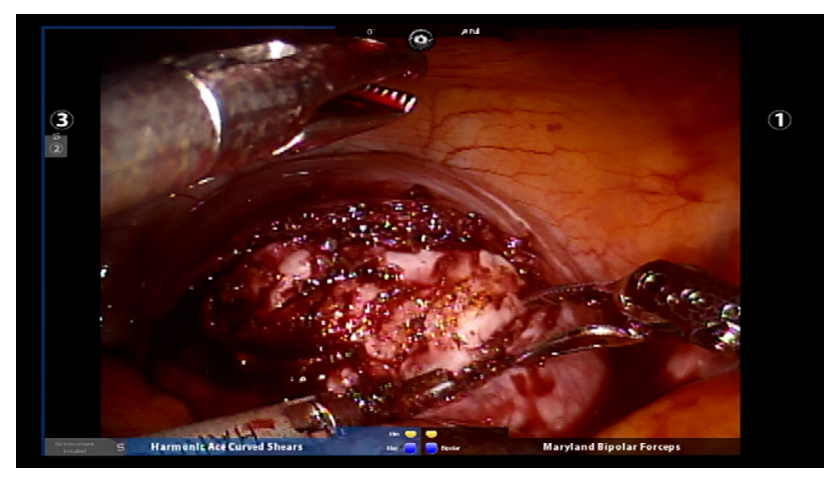

Figure 2 Hemostasis can be achieved using the Maryland bipolar electrocautery device while making the hysterotomy.

Note: Photo courtesy of Dr Tommaso Falcone, Department of Obstetrics, Gynecology, and Women's Health Institute, Cleveland Clinic, Cleveland, Ohio, USA. 
However, vasopressin is injected directly into the stalk of the fibroid. The fibroid stalk is then transected preferably by using the LigaSure device (Covidien). Deep intramural fibroids and fibroids located in the broad ligament are challenging to operate on robotically and require meticulous laparoscopic suturing skills. Advanced knowledge of the pelvic anatomy is also required in operating on these myomas, as the uterine blood vessels and the ureters can course adjacent to them. Once all of the myomas have been removed, the total number should be documented by the surgical team, in order to avoid any retained specimens inside the abdomen once the case is over. Small myomas can sometimes travel cephalad or roll behind the liver or under the bowel loops, and it is essential to keep track of their numbers. The next step is closure of the hysterotomy. Robotic surgery offers the benefit of ease and speed of suturing, which is important for limiting blood loss during the procedure. We typically prefer using a barbed suture such as V-Loc ${ }^{\text {TM }}$ by Covidien or Quill'TM SRS by Angiotech as with conventional laparoscopy. The advantage of this suture is that the surgeon is not required to tie a knot with this type of suture, and therefore closure of the

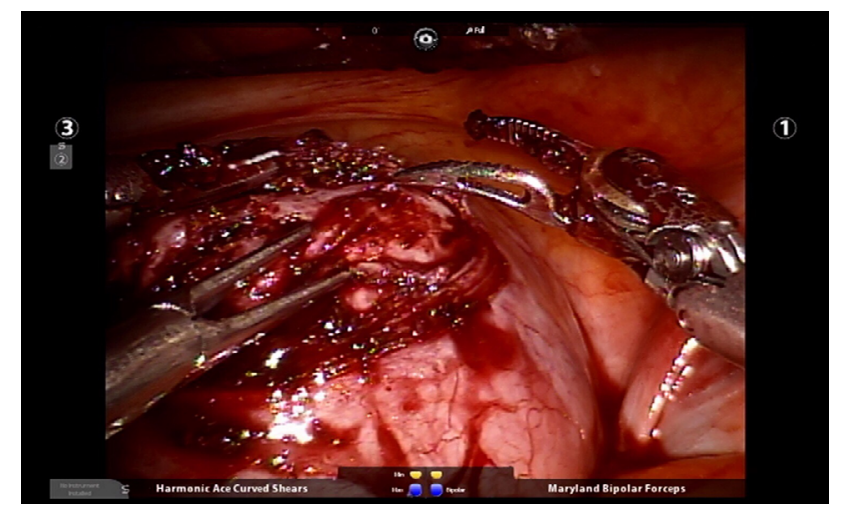

Figure 3 Placement of a robotic tenaculum on the fibroid to assist with enucleation of the fibroid.

Note: Photo courtesy of Dr Tommaso Falcone, Department of Obstetrics, Gynecology, and Women's Health Institute, Cleveland Clinic, Cleveland, Ohio, USA.

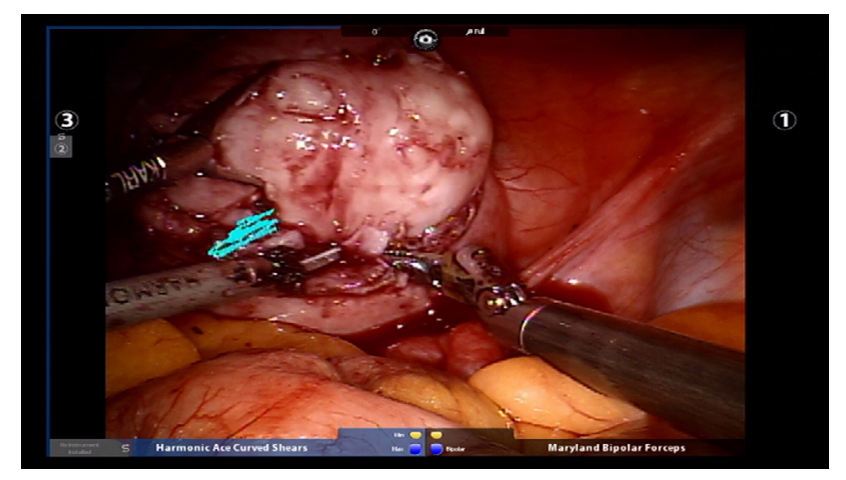

Figure 4 Fibroid enucleation with the assistance of robotic tenaculum using blunt dissection in conjunction with the use of harmonic energy as needed.

Note: Photo courtesy of Dr Tommaso Falcone, Department of Obstetrics, Gynecology, and Women's Health Institute, Cleveland Clinic, Cleveland, Ohio, USA. hystrotomy can be achieved more rapidly. The barbs also help with distributing the tension along the entire length of the suture without the need for applying constant tension while suturing, and therefore assist with maintaining hemostasis. Previous studies have demonstrated shorter suturing time and less intraoperative bleeding with the use of barbed suture in laparoscopic myomectomy. ${ }^{58}$ Another study by Einarsson et al reported a significant decrease in operative time and a shorter hospital stay when barbed suture was used for laparoscopic myomectomy compared to traditional suture. ${ }^{59}$ Given all the demonstrated advantages of the barbed suture in the literature, we contemplate the use of this suture for closure of the uterine defects. Closure of the hysterotomy robotically is similar to an open myomectomy and has to be performed in multiple layers. Adequate myometrial closure is an important issue to consider when translating open surgical techniques to robotic surgery. Closure of the deep layer is performed to close the dead space, followed by placement of additional suture layers to re-approximate the myometrial tissue and provide additional strength and hemostasis. After re-approximation of the uterine myometrium, the serosal layer is then closed using 2-0 or 3-0 absorbable monofilament polydioxanone suture in a running fashion. All of these sutures can be introduced and passed using the GelPoint. Hemostatic agents can be used as mentioned previously on the oozing areas.

The power morcellator was used commonly in the past for tissue removal at the end of myomectomy. However, the US Food and Drug Administration has imposed a moratorium on power morcellation or electromechanical morcellation for hysterectomy and myomectomy. This is mainly due to the potential risk for spreading malignancy in the case of an occult uterine cancer. Leiomyosarcoma is the most aggressive type of undiagnosed uterine malignancy with a poor prognosis and an approximate prevalence of 1 in 400 to 1 in 1000 women with a presumed benign uterine myoma. Due to this risk, we completely avoid the use of any power morcellation by using the suprapubic GelPoint device instead for tissue extraction. It is important, however, to note that the AAGL highlights that at this time there are not enough data to recommend against power morcellation in properly selected patients who are at low risk for the presence of a uterine or cervical malignancy or premalignancy. It is therefore necessary to appropriately assess surgical candidates preoperatively to determine whether the use of power morcellation may be an option. ${ }^{60,61}$

After complete removal of all the uterine fibroids, they are brought to the level of the suprapubic incision inside the GelPoint and either extracted intact or morcellated ex vivo 
using a scalpel. ${ }^{60,61}$ There is no evidence supporting that the use of these techniques will affect the prognosis of a leiomyosarcoma. After removal and extraction of all the fibroids, the robot can be undocked. After unlocking each of the arms from each trocar, the robot can be pulled away from the patient. The trocars are then removed under direct visualization and the fascia underlying the umbilical port is closed using a Carter-Thomason device (CooperSurgical). Skin closure is then performed, and the procedure is concluded.

\section{Postoperative care}

The principles of postoperative management after a robotic myomectomy are similar to those of a laparoscopic myomectomy. Early mobilization and pain control are the essential goals. Patient can be discharged later on the day of or the following day after surgery. Depending on the extent of the surgical procedure, disruption of the uterine myometrium, and entry into the endometrial cavity, the surgeon should have a conversation with the patient regarding obstetrics recommendations and potential need for future cesarean section. These recommendations should also be documented in the operative report by the surgeon for future review by the obstetrician. The potential increased risk of uterine rupture should also be discussed.

\section{Surgical complications}

Robotic myomectomy is considered to be a safe, minimally invasive alternative to open myomectomy for appropriately selected surgical candidates. It is associated with a shorter hospital stay compared to abdominal myomectomy. There is also improved cosmesis due to smaller skin incisions, less analgesic use, faster return to normal activities, and more rapid return of the bowel function. ${ }^{62}$ The costs, however, are higher with the use of this surgical technique given all the equipment expenses. Due to its minimally invasive nature, robotic myomectomy is associated with a very minimal complication profile. In our experience, robotic myomectomy results in less operative blood loss compared to conventional laparoscopic myomectomy and abdominal myomectomy. ${ }^{41}$ An advantage of robotic myomectomy is that it can be performed safely in patients with different body mass indices, and that obesity does not necessarily affect the surgical outcomes. ${ }^{63}$ Furthermore, robotic surgery can be performed without the need for steep Trendelenburg position, which may not be ideal in certain patients. ${ }^{64}$

\section{Surgical outcomes}

Robotic myomectomy is overall associated with many desirable outcomes compared to other types of myomectomy, especially abdominal myomectomy. A significant advantage of the robot technology is the opportunity to perform a minimally invasive surgery for myomectomy in patients who would have otherwise required a traditional laparotomy procedure for myomectomy. ${ }^{47}$ This advantage is due to the fact that the robot offers the surgeon the ability to apply open surgical techniques in a minimally invasive fashion. As previously mentioned and also from our center's experience, robotic myomectomy has been associated with decreased blood loss and length of hospital stay in comparison with other surgical techniques, albeit with an increased operative time and surgical costs. ${ }^{41}$

From a reproductive standpoint, studies have shown advantageous reproductive outcomes and high pregnancy rates in patients who have undergone robotic myomectomy ${ }^{65-68}$ The robot is capable of providing the surgeon with dexterity, thereby achieving a more gentle tissue dissection and handling, which can favor reproductive outcomes.

Some of the other benefits to the use of the surgical robot in gynecological surgery include the three-dimensional image of the operative field provided by the surgical console, the ease of suturing, improved and precise suture handling, the ability of the robotic endoscopic instruments to mimic the dexterity of the human hand to provide a greater range of motion and depth perception to increase surgical precision, and the motion scaling which eliminates tremor. The autonomous control over both camera and the instruments decreases surgical fatigue in contrast to complex laparoscopic and laparotomy cases, which may require the surgeon to contort his or her body in various positions for a long period of time. ${ }^{69}$ Robotic myomectomy also provides improved cosmetic results and is associated with improvements in postoperative pain outcomes as mentioned previously.

Long-term end points of robotic myomectomy including recurrence of myomas, quality of life outcomes, and obstetric outcomes are lacking and require additional data. These data will be important for determining the ultimate value and efficacy of robotic procedures.

\section{Limitations}

The da Vinci system is not without its own limitations. Some of the major limitations include surgical system and specialized equipment costs, the need for personnel training, the learning curve associated with learning a new surgical technique, and extended operative time. There is also a lack of tactile feedback during the procedure, which may lead to breakage of suture or applying excessive traction on the myoma resulting in breaching and compromising the endometrial cavity, which is an undesirable outcome. Port placement 
in robotic surgery is another limitation as the ports are larger than the typical conventional laparoscopic ports and they are placed higher on the abdomen. This higher port placement will make possible conversion of robotic to laparoscopic myomectomy more challenging if needed. The use of the robot system is also limited due to its size. Any position changing requires undocking and re-docking the robot which will result in even more added surgical time. ${ }^{62}$ These aspects can all have a great impact on the cost-effectiveness of robotic surgery.

\section{Conclusion}

The field of robotic surgery has made significant advancements over the last decade, and its application has become increasingly common in gynecological surgery. Gynecological procedures were reported to be comprising $\sim 60 \%$ of the total robotic surgeries performed in $2013 .{ }^{70}$ As the robot technology continues to grow, it is expected that additional revisions, technological advancements, and rapidly expanding modifications will be made to this system. Some of these potential future directions include the use of smaller robotic equipment, applying measures to decrease the set up and surgical time needed for the robot, including assisted docking, introducing single incision surgeries, and the ability to perform telesurgery using the robot system. These potential directions can provide the field of robotic surgery with an array of opportunities for clinical investigation. Additional prospective research studies are required to provide further information regarding long-term end points after robotic surgery and the cost effectiveness of this surgical technique.

\section{Disclosure}

The authors report no conflicts of interest in this paper.

\section{References}

1. Falcone T, Goldberg JM, Margossian H, Stevens L. Robotic-assisted laparoscopic microsurgical tubal anastomosis: a human pilot study. Fertil Steril. 2000;73(5):1040-1042.

2. Tan SJ, Lin CK, Fu PT, et al. Robotic surgery in complicated gynecologic diseases: experience of Tri-Service General Hospital in Taiwan. Taiwan J Obstet Gynecol. 2012;51(1):18-25.

3. SfakianosGP,FrederickPJ,KendrickJE, StraughnJM, KilgoreLC, HuhWK. Robotic surgery in gynecologic oncology fellowship programs in the USA: a survey of fellows and fellowship directors. Int J Med Robot. 2010;6(4):405-412.

4. Chiu LH, Chen CH, Tu PC, Chang CW, Yen YK, Liu WM. Comparison of robotic surgery and laparoscopy to perform total hysterectomy with pelvic adhesions or large uterus. J Minim Access Surg. 2015;11(1):87-93.

5. Palep JH. [Not Available]. J Minim Access Surg. 2009;5(1):1-7.

6. Nguan C, Girvan A, Luke PP. Robotic surgery versus laparoscopy; a comparison between two robotic systems and laparoscopy. $J$ Robot Surg. 2008;1(4):263-268.

7. Sinha R, Sanjay M, Rupa B, Kumari S. Robotic surgery in gynecology. J Minim Access Surg. 2015;11(1):50-59.
8. Wright JD, Herzog TJ, Tsui J, Ananth CV, Lewin SN, Lu YS, Neugut AI, Hershman DL. Nationwide trends in the performance of inpatient hysterectomy in the United States. Obstet Gynecol. 2013;122(2 Pt 1): 233-241.

9. Liu H, Lawrie TA, Lu D, Song H, Wang L, Shi G. Robot-assisted surgery in gynaecology. Cochrane Database Syst Rev. 2014;(12):CD011422.

10. Bloski T, Pierson R. Endometriosis and chronic pelvic pain: unraveling the mystery behind this complex condition. Nurs Womens Health. 2008;12(5):382-395.

11. Nezhat C, Lewis M, Kotikela S, et al. Robotic versus standard laparoscopy for the treatment of endometriosis. Fertil Steril. 2010;94(7): 2758-2760.

12. Neme RM, Schraibman V, Okazaki S, et al. Deep infiltrating colorectal endometriosis treated with robotic-assisted rectosigmoidectomy. JSLS. 2013;17(2):227-234.

13. Sotrel G. Is surgical repair of the fallopian tubes ever appropriate? Rev Obstet Gynecol. 2009;2(3):176-185.

14. Rodgers AK, Goldberg JM, Hammel JP, Falcone T. Tubal anastomosis by robotic compared with outpatient minilaparotomy. Obstet Gynecol. 2007;109(6):1375-1380.

15. Dharia Patel SP, Steinkampf MP, Whitten SJ, Malizia BA. Robotic tubal anastomosis: surgical technique and cost effectiveness. Fertil Steril. 2008;90(4):1175-1179.

16. Sparic R, Mirkovic L, Malvasi A, Tinelli A. Epidemiology of uterine myomas: a review. Int J Fertil Steril. 2016;9(4):424-435.

17. Drayer SM, Catherino WH. Prevalence, morbidity, and current medical management of uterine leiomyomas. Int J Gynaecol Obstet. 2015; 131(2):117-122.

18. Kashani BN, Centini G, Morelli SS, Weiss G, Petraglia F. Role of medical management for uterine leiomyomas. Best Pract Res Clin Obstet Gynaecol. 2016;34:85-103.

19. Gurusamy KS, Vaughan J, Fraser IS, Best LM, Richards T. Medical therapies for uterine fibroids - a systematic review and network meta-analysis of randomised controlled trials. PLoS One. 2016;11(2):e0149631.

20. Donnez J, Dolmans MM. Uterine fibroid management: from the present to the future. Hum Reprod Update. 2016;22(6):665-686.

21. de Bruijn AM, Ankum WM, Reekers JA, et al. Uterine artery embolization vs hysterectomy in the treatment of symptomatic uterine fibroids: 10-year outcomes from the randomized EMMY trial. Am J Obstet Gynecol. 2016;215(6):745e1-745e12.

22. Zepiridis LI, Grimbizis GF, Tarlatzis BC. Infertility and uterine fibroids. Best Pract Res Clin Obstet Gynaecol. 2016;34:66-73.

23. Practice Committee of American Society for Reproductive Medicine in collaboration with Society of Reproductive Surgeons. Myomas and reproductive function. Fertil Steril. 2008;90(5 Suppl):S125-S130.

24. Ciavattini A, Tsiroglou D, Litta P, Frizzo H, Tranquilli AL. Ultra-minilaparotomy myomectomy: a minimally invasive surgical approach for the treatment of large uterine myomas. Gynecol Obstet Invest. 2009;68(2): 127-133.

25. Mukhopadhaya N, De Silva C, Manyonda IT. Conventional myomectomy. Best Pract Res Clin Obstet Gynaecol. 2008;22(4):677-705.

26. Mcllveen M, Li TC. Myomectomy: a review of surgical technique. Hum Fertil (Camb). 2005;8(1):27-33.

27. Wong LF, Gleeson N. Myomectomy: a suturing technique for the open procedure. J Obstet Gynaecol. 2013;33(2):197-198.

28. Taylor A, Sharma M, Tsirkas P, Di Spiezio Sardo A, Setchell M, Magos A. Reducing blood loss at open myomectomy using triple tourniquets: a randomised controlled trial. BJOG. 2005;112(3):340-345.

29. Conforti A, Mollo A, Alviggi C, et al. Techniques to reduce blood loss during open myomectomy: a qualitative review of literature. Eur $J$ Obstet Gynecol Reprod Biol. 2015;192:90-95.

30. Guarnaccia MM, Rein MS. Traditional surgical approaches to uterine fibroids: abdominal myomectomy and hysterectomy. Clin Obstet Gynecol. 2001;44(2):385-400.

31. Sami Walid M, Heaton RL. The role of laparoscopic myomectomy in the management of uterine fibroids. Curr Opin Obstet Gynecol. 2011;23(4):273-277. 
32. Sinha R, Hegde A, Mahajan C, Dubey N, Sundaram M. Laparoscopic myomectomy: do size, number, and location of the myomas form limiting factors for laparoscopic myomectomy? J Minim Invasive Gynecol. 2008;15(3):292-300.

33. Mattei A, Cioni R, Bargelli G, Scarselli G. Techniques of laparoscopic myomectomy. Reprod Biomed Online. 2011;23(1):34-39.

34. Chittawar PB, Kamath MS. Review of nonsurgical/minimally invasive treatments and open myomectomy for uterine fibroids. Curr Opin Obstet Gynecol. 2015;27(6):391-397.

35. Holzer A, Jirecek ST, Illievich UM, Huber J, Wenzl RJ. Laparoscopic versus open myomectomy: a double-blind study to evaluate postoperative pain. Anesth Analg. 2006;102(5):1480-1484.

36. Cicinelli E, Tinelli R, Colafiglio G, Saliani N. Laparoscopy vs minilaparotomy in women with symptomatic uterine myomas: a prospective randomized study. J Minim Invasive Gynecol. 2009;16(4):422-426.

37. Jin $\mathrm{C}, \mathrm{Hu} \mathrm{Y}$, Chen $\mathrm{XC}$, et al. Laparoscopic versus open myomectomy - a meta-analysis of randomized controlled trials. Eur J Obstet Gynecol Reprod Biol. 2009;145(1):14-21.

38. Chang CC, Chen W. A comparison of surgical outcomes between laparoscopic and open myomectomy in Southern Taiwan. Int J Gynaecol Obstet. 2012;119(2):189-193.

39. Chang CC. A comparison of the costs of laparoscopic myomectomy and open myomectomy at a teaching hospital in southern Taiwan. Taiwan J Obstet Gynecol. 2013;52(2):227-232.

40. Bhave Chittawar P, Franik S, Pouwer AW, Farquhar C. Minimally invasive surgical techniques versus open myomectomy for uterine fibroids. Cochrane Database Syst Rev. 2014;(10):CD004638.

41. Iavazzo C, Mamais I, Gkegkes ID. Robotic assisted vs laparoscopic and/or open myomectomy: systematic review and meta-analysis of the clinical evidence. Arch Gynecol Obstet. 2016;294(1):5-17.

42. Bedient CE, Magrina JF, Noble BN, Kho RM. Comparison of robotic and laparoscopic myomectomy. Am J Obstet Gynecol. 2009;201(6):566. e1-e5.

43. Nezhat C, Lavie O, Hsu S, Watson J, Barnett O, Lemyre M. Roboticassisted laparoscopic myomectomy compared with standard laparoscopic myomectomy - a retrospective matched control study. Fertil Steril. 2009;91(2):556-559.

44. Soto E, Flyckt R, Falcone T. Endoscopic management of uterine fibroids: an update. Minerva Ginecol. 2012;64(6):507-520.

45. Seracchioli R, Rossi S, Govoni F, et al. Fertility and obstetric outcome after laparoscopic myomectomy of large myomata: a randomized comparison with abdominal myomectomy. Hum Reprod. 2000;15(12): 2663-2668.

46. Soto E, Flyckt R, Falcone T. Minimally invasive myomectomy using unidirectional knotless barbed suture. JMinim Invasive Gynecol. 2014;21(1):27.

47. Dubuisson JB, Fauconnier A, Fourchotte V, Babaki-Fard K, Coste J, Chapron C. Laparoscopic myomectomy: predicting the risk of conversion to an open procedure. Hum Reprod. 2001;16(8):1726-1731.

48. Crosignani PG, Vercellini P, Meschìa M, Oldani S, Bramante T. GnRH agonists before surgery for uterine leiomyomas. A review. J Reprod Med. 1996;41(6):415-421.

49. Moghadam R, Lathi RB, Shahmohamady B, et al. Predictive value of magnetic resonance imaging in differentiating between leiomyoma and adenomyosis. JSLS. 2006;10(2):216-219.

50. Tulandi T, Béique F, Kimia M. Pulmonary edema: a complication of local injection of vasopressin at laparoscopy. Fertil Steril. 1996;66(3):478-480.

51. Hobo R, Netsu S, Koyasu Y, Tsutsumi O. Bradycardia and cardiac arrest caused by intramyometrial injection of vasopressin during a laparoscopically assisted myomectomy. Obstet Gynecol. 2009;113(2 Pt 2):484-486.
52. Hung MH, Wang YM, Chia YY, Chou YM, Liu K. Intramyometrial injection of vasopressin causes bradycardia and cardiac arrest - report of two cases. Acta Anaesthesiol Taiwan. 2006;44(4):243-247.

53. Nezhat F, Admon D, Nezhat CH, Dicorpo JE, Nezhat C. Life-threatening hypotension after vasopressin injection during operative laparoscopy, followed by uneventful repeat laparoscopy. J Am Assoc Gynecol Laparosc. 1994;2(1):83-86.

54. Kongnyuy EJ, Wiysonge CS. Interventions to reduce haemorrhage during myomectomy for fibroids. Cochrane Database Syst Rev. 2014;(8):CD005355.

55. Litta $\mathrm{P}$, Fantinato S, Calonaci F, et al. A randomized controlled study comparing harmonic versus electrosurgery in laparoscopic myomectomy. Fertil Steril. 2010;94(5):1882-1886.

56. Raga F, Sanz-Cortes M, Bonilla F, Casañ EM, Bonilla-Musoles F. Reducing blood loss at myomectomy with use of a gelatin-thrombin matrix hemostatic sealant. Fertil Steril. 2009;92(1):356-360.

57. Alessandri F, Remorgida V, Venturini PL, Ferrero S. Unidirectional barbed suture versus continuous suture with intracorporeal knots in laparoscopic myomectomy: a randomized study. J Minim Invasive Gynecol. 2010;17(6):725-729.

58. Einarsson J, ChavanNR, SuzukiY, JonsdottirG, VellingaTT, Greenberg JA. Use of bidirectional barbed suture in laparoscopic myomectomy: evaluation of perioperative outcomes, safety, and efficacy. J Minim Invasive Gynecol. 2011;18(1):92-95.

59. Brown J. AAGL advancing minimally invasive gynecology worldwide: statement to the FDA on power morcellation. J Minim Invasive Gynecol. 2014;21(6):970-971.

60. AAGL Advancing Minimally Invasive Gynecology Worldwide. AAGL practice report: morcellation during uterine tissue extraction. J Minim Invasive Gynecol. 2014;21(4):517-530.

61. Catenacci M, Flyckt RL, Falcone T. Robotics in reproductive surgery: strengths and limitations. Placenta. 2011;32(Suppl 3):S232-S237.

62. Barakat EE, Bedaiwy MA, Zimberg S, Nutter B, Nosseir M, Falcone T. Robotic-assisted, laparoscopic, and abdominal myomectomy: a comparison of surgical outcomes. Obstet Gynecol. 2011;117(2 Pt 1): $256-265$.

63. George A, Eisenstein D, Wegienka G. Analysis of the impact of body mass index on the surgical outcomes after robot-assisted laparoscopic myomectomy. J Minim Invasive Gynecol. 2009;16(6): $730-733$.

64. Ghomi A, Kramer C, Askari R, Chavan NR, Einarsson JI. Trendelenburg position in gynecologic robotic-assisted surgery. J Minim Invasive Gynecol. 2012;19(4):485-489.

65. Lönnerfors C, Persson J. Robot-assisted laparoscopic myomectomy: a feasible technique for removal of unfavorably localized myomas. Acta Obstet Gynecol Scand. 2009;88(9):994-999.

66. Bocca S, Stadtmauer L, Oehninger S. Uncomplicated full term pregnancy after da Vinci-assisted laparoscopic myomectomy. Reprod Biomed Online. 2007;14(2):246-249.

67. Cela V, Freschi L, Simi G, et al. Fertility and endocrine outcome after robot-assisted laparoscopic myomectomy (RALM). Gynecol Endocrinol. 2013;29(1):79-82.

68. Lönnerfors C, Persson J. Pregnancy following robot-assisted laparoscopic myomectomy in women with deep intramural myomas. Acta Obstet Gynecol Scand. 2011;90(9):972-977.

69. Hickman LC, Kotlyar A, Luu TH, Falcone T. Do we need a robot in endometriosis surgery? Minerva Ginecol. 2016;68(3):380-387.

70. Xie Y. Cost-effectiveness of robotic surgery in gynecologic oncology. Curr Opin Obstet Gynecol. 2015;27(1):73-76. 


\section{Publish your work in this journal}

Robotic Surgery: Research and Reviews is an international, peer reviewed, open access, online journal publishing original research, commentaries, reports, and reviews on the theory, use and application of robotics in surgical interventions. Articles on the use of supervisory-controlled robotic systems, telesurgical devices, and shared-control systems are invited. The manuscript management system is completely online and includes a very quick and fair peer review system, which is all easy to use. Visit http://www.dovepress.com/testimonials.php to read real quotes from published authors.

Submit your manuscript here: https://www.dovepress.com/robotic-surgery-research-and-reviews-journal 